\title{
EDITORIAL
}

\section{Immune system dysregulation in chronic lung disease}

\author{
B.J. Marsland*,\#, M. Königshoff', S. Saglani ${ }^{+}$and O. Eickelberg
}

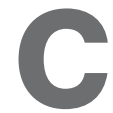
hronic lung diseases (CLDs), including chronic obstructive pulmonary disease (COPD), asthma, lung cancer, interstitial lung disease and pulmonary hypertension, present a significant and rising global health problem. CLDs develop at the interface between genetic susceptibilities and environmental aspects, and are currently the second leading cause of death worldwide. Thus far, therapies of only limited effect exist for CLDs; the search for novel and effective therapeutic strategies has been at the centre of recent major research efforts. As part of the pathogenesis of many CLDs, ill-defined lung injuries and failed repair mechanisms ultimately lead to perturbed lung architecture that is incompatible with normal respiratory function. In particular, the immunological mechanisms that initiate and maintain CLD remain to be fully unravelled.

The mission of the European Respiratory Society (ERS) is to alleviate suffering from respiratory disease and promote lung health through research, knowledge sharing, and medical and public education. In support of this mission, the ERS holds an annual Lung Science Conference (LSC), which brings together leading basic scientists with physician scientists and clinicians on distinct topics that are of relevance to respiratory medicine.

In April 2011, world-leading scientists and clinicians met at the ninth LSC in Estoril, Portugal, to share their latest discoveries and to work towards identifying the cellular and molecular pathways that underlie the development of CLDs. The meeting highlighted major novel findings in immune system (dys)function in the major CLDs, as outlined in detail below. Some of the presentations discussed can be found in the E-Resources on the ERS website (at http://www.ers-education.org/pages/default. aspx?id=2556).

\section{CURRENT STATUS OF ASTHMA RESEARCH: ATOPY AND THE EPITHELIUM}

The incidence of asthma has been steadily increasing in recent decades and intensive research has particularly focused upon the role of T-helper cell (Th) type 2 responses and therapies designed to neutralise the activity of Th2-associated cytokines, such as interleukin (IL)-4, IL-5 and IL-13. Disappointing results from clinical trials have suggested that, although these cells and cytokines were strongly associated with allergic disease,

\footnotetext{
* Service de Pneumologie, Centre Hospitalier Universitaire Vaudois and ${ }^{\#}$ Faculty of Biology and Medicine, University of Lausanne Vaudois Lausanne, Lausanne, Switzerland. "Comprehensive Pneumology Center, Ludwig-Maximilians-University, University Hospital Grosshadern, and Helmholtz Zentrum München, Munich, Germany. ${ }^{+}$Respiratory Paediatrics, Royal Brompton Hospital, Imperial College London, London, UK.
}

CORRESPONDENCE: O. Eickelberg, Comprehensive Pneumology Center, Ludwig Maximilians University Munich, University Hospital Grosshadern, and Helmholtz Zentrum München, MaxLebsche-Platz 31, 81377 München, Germany. E-mail: oliver.eickelberg@helmholtz-muenchen.de blocking and/or neutralising them alone was insufficient to substantially ameliorate or prevent asthma. In a keynote presentation, Prof. Stephen Holgate (University of Southampton, Southampton, UK) highlighted the airway epithelium as a central determinant for the development and pathogenesis of asthma, as opposed to Th2 responses per se. Notably, large-scale approaches, such as genome wide association studies (GWAS), have recently uncovered disease-relevant genes in asthma, which are associated with airway epithelial cell function rather than Th2 responses. Indeed, key components of the allergic disease might be genes associated with maintenance of the epithelial barrier integrity (e.g. tight junctions), innate immunity or epithelial growth factors. At its core, the chronicity of asthma may thus be due to defective epithelial cell responses, integrity and repair, compounded by inappropriate inflammatory responses against innocuous environmental allergens. With this in mind, the most effective antiasthma therapies of the future may be those targeting pathways associated with epithelial cell layer integrity and repair.

Prof. Sebastian Johnston (Imperial College London, London, UK) highlighted data revealing that production of interferons was impaired during rhinovirus-induced exacerbations of asthma. Importantly, these data suggest the use of interferons as a candidate new therapy for asthma exacerbations. Following the line of key cellular players in asthma, Prof. Clare Lloyd (Imperial College London) presented evidence for the importance of IL-17 in $\gamma \delta$ T-cell responses during asthma.

\section{EARLY ORIGINS OF CLDS}

There is growing evidence that events in the first years of life can influence susceptibility to disease. Prof. Patrick Holt (Telethon Institute for Child Health Research, Perth, Australia) outlined that early respiratory infections are one of the strongest associations with the development of asthma. Prof. Holt argued that a central determinant of increased persistent asthma is the concurrent exposure to allergens and viral infection. Support for this perspective is found in mouse models of disease, which have shown that concurrent viral infections can break tolerance of aero-allergens or indeed enhance their presentation to Th2 cells by increasing dendritic cell activation and migration.

In recent work by the laboratory of Prof. Charles Mackay (Monash University, Melbourne, Australia), a link between diet and inflammation was attributed to the cellular receptors GPR41 and 43. These G-protein-coupled receptors recognise shortchain fatty acids (SCFA), microbial gastrointestinal-derived metabolites of fibre. Mice lacking these receptors on their cells, which are unable to respond to SCFA, exhibit an exaggerated disease development in models of arthritis, colitis and allergic airway inflammation. Removing the microbiota (in axenic/ germ-free mice) and consequently reducing the circulating 
levels of SCFA exaggerated disease development, while increasing the levels of SCFA by administration of exogenous SCFA-suppressed disease. These findings strongly link dietary intake with inflammation and it is tempting to speculate that diets high in fibre may be protective against chronic inflammation. Moreover, changes in diet in the Western world might underlie the trend in increasing incidence of allergy and autoimmunity over recent decades.

It appears clear that there will be multiple pathways through which early childhood events could impact upon the development of lung inflammation. Prof. Troy Randall (University of Rochester Medical Center, Rochester, NY, USA) presented latebreaking data, indicating that there is a window of development in neonatal mice, in which there is an increased propensity towards the development of inducible broncho-associated lymphoid tissue (iBALT). Specifically, administration of lipopolysaccharide (LPS) to the lungs of mice within the first weeks of birth led to an IL-17-dependent increase in iBALT, which, strikingly, was maintained at a high level through adulthood. The presence of iBALT indicates that adaptive immune responses in the lung might develop locally or more rapidly following antigen inhalation; such enhanced formation due to early childhood exposures could act to shape inflammation later in life. Whether such local responses would be protective (by enhancing the response against viral infection, for example) or could in fact sustain allergic responses or tissue damage, remains to be seen.

\section{MECHANISMS UNDERLYING COPD AND FIBROSIS}

Accurate detection of the early stages of COPD/emphysema has proven difficult. Employing cutting-edge pathological techniques, Prof. James Hogg and colleagues (University of British Columbia, Vancouver, BC, Canada) have made substantial contributions to our understanding of this disease. Indeed, detailed examination of human COPD lung tissue indicates that a narrowing and destruction of the terminal bronchioles (small airway remodelling and fibrosis) precedes emphysema formation. Prof. Hogg's data also indicate that macrophages, rather than neutrophils, were predominantly present in emphysematous tissues; whether this provides clues to the critical cells involved in the maintenance of chronicity, tissue damage or repair remains to be elucidated.

Unquestionably, macrophages are strongly linked with COPD, as discussed by Prof. Peter Barnes (Imperial College London). Indeed, there is a $5-10$-fold increase in the number of macrophages in the airways and parenchyma of COPD patients, which increases in line with the severity of emphysema. Data indicates that macrophages isolated from COPD patients release higher levels of inflammatory mediators, such as chemokines, cytokines and proteases, whilst having a reduced capacity to phagocytose particles. Moreover, although corticosteroids are highly effective at controlling inflammation in general, they are ineffective in certain cases of COPD, as macrophages from COPD patients can be insensitive to corticosteroids. The exact role of macrophages in COPD needs to be clarified; specifically, whether macrophages are acting as instigators of disease or maintainers of chronicity, or whether they are simply trying to clear up the damage.

Neutrophils are another major cell type associated with COPD. In particular, neutrophil elastase (NE) has been shown to influence cigarette smoke-induced emphysema. At the 2011 LSC, Prof. Steven Shapiro (University of Pittsburgh, Pittsburgh, PA, USA) presented that mice deficient in NE also exhibited decreased monocyte recruitment. The role of neutrophils and NE might in fact be less related to the direct protease activity damaging the lung tissue, and more related to the need for NE for neutrophil migration into tissues and recruitment of alternate cell types, such as monocytes. The balance between neutrophil and macrophage recruitment, clearance and effector function, remains at the core of COPD pathogenesis.

Lung fibrosis is regulated by several cytokines, chemokines and growth factors. Transforming growth factor- $\beta$ is a potent pro-fibrotic mediator and is involved in IL-17A production by lymphocytes. In his presentation, Prof. Thomas Wynn (National Institute of Allergy and Infectious Diseases, Bethesda, MD, USA) discussed the impact of IL-17A-driven inflammation within the development of bleomycin-induced fibrosis, while pointing out that IL-13 is critically involved in Schistosoma mansoni-driven fibrosis. IL-17A is a key mediator of bleomycininduced fibrosis driven by transforming growth factor- $\beta$, as well as IL-1 $\beta$. Importantly, he highlighted that these distinct immune responses may be critical to the nature of the remodelling processes and crucial to the identification of specific therapeutic strategies.

Ultimately, many CLD may be relieved if we understood how to regenerate normal lung tissue in the adult human, perhaps through tissue-engineering approaches or stem cell biology. In this respect, Prof. Marc Humbert (INSERM, Paris, France) has highlighted c-kit-positive cells as putative progenitor cells homing to the perivascular compartment in pulmonary arterial hypertension, possibly contributing to the vascular remodelling process characteristic of this condition.

It will be this focus on lung regeneration that we will highlight during the 10th LSC in 2012. Therefore, we invite you to join us for the "Rebuilding a diseased lung: repair and regeneration" LSC, from March 30 to April 1, 2012, in Estoril, Portugal.

\section{STATEMENT OF INTEREST}

A statement of interest for O. Eickelberg can be found at www.erj. ersjournals.com/site/misc/statements.xhtml 